\title{
Preparation of silver nanoparticle loaded cotton threads to facilitate measurement development for textile applications
}

Version 1.0

J. M. Gorham

K. Murphy

J. Liu

D. Tselenchuk

G. Stan

T. M. Nguyen

R. D. Holbrook

M. Winchester

R. F. Cook

R. I. MacCuspie

V. A. Hackley 


\title{
Preparation of silver nanoparticle loaded cotton threads to facilitate measurement development for textile applications
}

\author{
Version 1.0 \\ J. M. Gorham \\ J. Liu \\ D. Tselenchuk \\ G. Stan \\ T. M. Nguyen \\ R. D. Holbrook \\ V. A. Hackley \\ R. F. Cook \\ Material Measurement Science Division \\ Material Measurement Laboratory \\ K. Murphy \\ M. Winchester \\ Chemical Sciences Division \\ Material Measurement Laboratory \\ R. I. MacCuspie \\ Florida Polytechnic University \\ Lakeland, FL
}

This publication is available free of charge from:

http://dx.doi.org/10.6028/NIST.SP.1200-8

January 2015

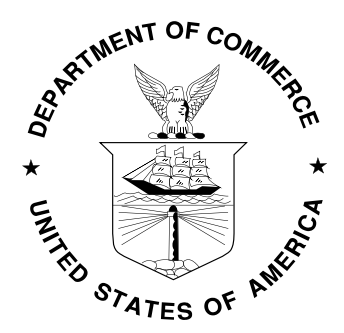

U.S. Department of Commerce

Penny Pritzker, Secretary

National Institute of Standards and Technology Willie May, Acting Under Secretary of Commerce for Standards and Technology and Acting Director 
Certain commercial entities, equipment or materials may be identified in this document in order to describe an experimental procedure or concept adequately. Such identification is not intended to imply recommendation or endorsement by the National Institute of Standards and Technology, nor is it intended to imply that the entities, materials or equipment are necessarily the best available for the purpose.

National Institute of Standards and Technology Special Publication 1200-8

Natl. Inst. Stand. Technol. Spec. Publ. 1200-8, 21 pages (January 2015)

CODEN: NSPUE2

This publication is available free of charge from:

http://dx.doi.org/10.6028/NIST.SP.1200-8 


\section{FOREWORD}

This NIST Special Publication (SP) is one in a series of NIST SPs that address research needs articulated in the National Nanotechnology Initiative (NNI) Environmental, Health, and Safety Research Strategy published in 2011 [1]. This Strategy identified a Nanomaterial Measurement Infrastructure (NMI) as essential for science-based risk assessment and risk management of nanotechnology-enabled products as pertaining to human health, exposure, and the environment. NIST was identified as the lead federal agency in the NMI core research area of the Strategy. This research area includes development of measurement tools for the detection and characterization of engineered nanomaterials in nanotechnology-enabled products. Textiles containing silver nanoparticles, the focus of this SP, are consumer products of great interest for nanotechnology environmental, health, and safety (nano-EHS) research and for product regulation.

The protocol presented in this SP describes a strategy for developing a validation known to contain silver nanoparticles loaded onto the surface of a cotton thread. These validation threads can be used to test the capabilities of various analytical methods to characterize silver nanoparticles in textiles. Updates to this protocol may be released in the future. Visit http://nist.gov/mml/np-measurement-protocols.cfm to check for revisions of this protocol or for new protocols in the series. We also encourage users of this protocol to reference it in their publications. 


\section{Introduction}

Silver (Ag) has long been used for its antiseptic qualities. These qualities are attributed to the surface oxidation of metallic $\mathrm{Ag}$ to $\mathrm{Ag}(+1)$ and a ligand $\left(\mathrm{Ag}^{0} \rightarrow \mathrm{Ag}(\mathrm{I}) \mathrm{L}\right)$ followed by the generation of an ion $\left(\mathrm{Ag}^{+}\right)$which can be toxic to many bacteria and fungi. Efforts to exploit this process have led to the widespread incorporation of Ag nanoparticles (AgNPs) into many consumer products as an anti-microbial agent. Their small size and large surface area to volume $(\mathrm{SA} / \mathrm{V})$ ratio result in increased rates of oxidation and subsequent dissolution compared to largerscale forms of $\mathrm{Ag}$. The enhancement of $\mathrm{Ag}^{+}$formation due to increased $\mathrm{SA} / \mathrm{V}$ ratios has led to the incorporation of AgNPs into composites and textiles with the aim of enhancing their antimicrobial efficacy. As highlighted in a recent review [2], the widespread usage of AgNPs has led to increased concerns regarding risk for humans and the environment. In the NNI 2011 EHS Research Strategy [1], risk is broadly defined as the product of hazard $\times$ exposure. In order to address the second component of this equation, a set of instrumentation must first be identified and proven to be capable of detecting AgNPs in consumer products and when released from products by testing controls known to have an AgNP component. Such an assessment is necessary to determine the potential for human or environmental exposure to AgNPs from clothing, bandages, toys, towels, etc. This SP provides a well-defined protocol for the generation of a control material to aid in the assessment of an instrument's measurement capabilities for detecting AgNP in textiles.

There are two approaches for assessing AgNP characteristics and potential for transformation due to their surroundings. The first focuses on the AgNPs and examines their formation and stability in various environments [3-5], using the information learned towards predicting likely behavior in applications after incorporation into a material or product. The scientific understanding gained from studying the formation and stability of AgNPs in various environments can be used to provide engineering guidance for the safe storage and handling of AgNP suspensions [4] and for the development of AgNP reference materials [1, 5] for instrument calibration or measurement intercomparison. The second approach focuses on AgNP -containing products, such as textiles, and examines the release of Ag in various environments [6], using the information learned towards assessing the binding strength of Ag to a textile. The engineering understanding gained from this approach can provide guidance for product handling and scientific insights into how AgNPs could be treated to control binding and release.

Researchers at NIST have followed both of these approaches. However, attempts to characterize and quantify specific properties of AgNP-containing textile products accurately (e.g., particle size, shape, number density, total Ag concentration, oxidation state) have been hindered by unknown variables in the products including the material identity of textile fibers, size of the nano-particulate Ag added to the textile, and the heterogeneity of the Ag within the consumer products. Furthermore, the presence of an actual "nanoparticle" form of Ag within these products was often unclear.

This SP provides a tool to evaluate an instruments effectiveness in characterizing AgNPs within consumer products that has been designed from aspects of the two aforementioned approaches. Specifically, a protocol for the preparation of a test material, more relevant than free AgNP suspensions, less complex than a Ag-containing commercial textile product and known to have 
an AgNP component, has been developed. AgNPs for the test material are generated by a solution-based reduction technique while cotton threads are fully immersed in the solution. The threads are then examined using analytical techniques typically employed by researchers to characterize AgNPs [3,4,6].

\section{Principles and Scope}

The chemical reduction of $\mathrm{Ag}^{+}$to form AgNPs stabilized with citrate is a commonly utilized synthesis method designed to make a relatively uniform suspensions of particles in water, typically with a particle diameter near $20 \mathrm{~nm}[3,4]$. These suspensions are employed in studies involving stability, fate and transport as well as in further processing to yield more chemically complex systems. This SP details a protocol where $\mathrm{Ag}^{+}$reduction is used for the in-situ loading of citrate-capped AgNPs onto the surface of cotton threads. This protocol provides a step-wise method to form AgNP for use in developing validation samples for determining the effectiveness of various analytical techniques commonly used in the characterization of consumer products containing AgNPs. The current protocol describes the preparation of AgNP loaded cotton threads: a baseline (control) thread containing no Ag and five threads containing progressively higher levels of Ag loading. In the remainder of the SP, validation samples were characterized by a suite of measurement techniques.

\section{Terminology}

Nanoscale: Characterized by a dimensions between approximately $1 \mathrm{~nm}$ and $100 \mathrm{~nm}$ [7]

Engineered nanomaterial: Materials that have been purposely synthesized or manufactured to have at least one external dimension of approximately 1 to 100 nanometers $(\mathrm{nm})$ and that exhibit unique properties determined by this size [1]

Nanoparticle: Sub-classification of ultrafine particle that is characterized by at least two dimensions in the nanoscale [7]; also referred to as "nano-object" [8]

Textile: Flexible woven material consisting of a network of natural or artificial fibers often referred to as thread or yarn [9]

Loading: Incorporation of Ag-NPs into cotton threads via a chemical solution-based method

\section{Materials and equipment}

\subsection{Starting materials}

4.1.1 12 sections ( $\approx 56 \mathrm{~cm}$ each) of white cotton thread ${ }^{1}$ (Gutermann). Each thread consists of a number of fibers.

4.1.2 Deionized (DI) water source.

4.1.3 Silver nitrate powder (0.36 g) (>99\% purity, Sigma Aldrich).

4.1.4 Trisodium citrate dihydrate powder (0.48 g) (Sigma Aldrich, meets US Pharmacopeia testing specifications).

4.1.5 Sodium borohydride powder (0.40 g) (99.99\% purity, Sigma Aldrich).

\footnotetext{
${ }^{1}$ The total number of threads may vary depending on the number of loadings and controls
} 
4.2 Glassware and standard equipment

4.2.1 6 cleaned $^{2}$ medium graduated borosilicate glass beakers $(\approx 250 \mathrm{ml}$ capacity).

4.2.2 6 cleaned Teflon stir bars.

4.2.3 3 cleaned small borosilicate glass beakers ( $50 \mathrm{~mL}$ to $100 \mathrm{~mL}$ capacity).

4.2.4 6 heated stir plates ${ }^{1}$.

4.2.5 Aluminum foil ${ }^{3}$.

4.2.6 Cleaned glass covers ${ }^{2,3}$.

4.2.7 2 cleaned large beakers $(>500 \mathrm{~mL})$.

\subsection{Measurement Equipment}

4.3.1 Analytical balance and plastic weigh boats.

4.3.2 Thermometer ${ }^{3}$.

4.3.3 A $10 \mathrm{~mL}$ and a $100 \mathrm{~mL}$ graduated cylinder.

4.3.4 Calibrated Eppendorf pipettes (capacity $10 \mu \mathrm{L}$ to $10 \mathrm{~mL})^{1}$.

4.3.5 Characterization instruments ${ }^{4}$.

4.3.5.1 Atomic Force Microscopy (AFM): Images were acquired using MultiMode Bruker (Santa Barbara/California) AFM equipped with PPP-SEIH integrated silicon probes from NanoSensors (Neuchatel/Switzerland).

4.3.5.2 Field Emission Scanning Electron Microscopy (FE-SEM): Images were acquired using a Hitachi S-4700, operated at an acceleration voltage of $3 \mathrm{keV}$. Sputtering was performed with a Denton Vacuum Desktop III system.

4.3.5.3 Energy Dispersive X-ray Spectroscopy (EDS): Quanta 200 environmental SEM (ESEM) from FEI (Hillsboro, OR)

4.3.5.4 Inductively Coupled Plasma-Mass Spectrometry (ICP-MS): Mass spectrometric analyses were performed on a ThermoFisher Scientific X series II ICP-MS.

4.3.5.5 X-ray Photoelectron Spectroscopy (XPS): XPS spectra were acquired on an Axis Ultra DLD spectrophotometer (Kratos Analytical, Chestnut Ridge, New York).

\section{Preparation and loading of AgNPs onto cotton threads}

5.1 Immerse the sections of cotton thread in DI water in a large beaker to wash off any contaminants, salts and/or small particulates that are loosely bound to the surface.

5.2 Label the six beakers with numbers 1 to 6 . These numbers correspond to the validated thread numbers in Table 1. Note that sample 6 is the control thread that contains no Ag. For these preparations, the control was prepared on a separate day.

5.3 Fill each medium beaker with $\approx 150 \mathrm{~mL}$ of DI water and place them on a heated stir plate. Into each beaker, add a stir bar and spin at a rate where a vortex is visible in the water. Heat

\footnotetext{
${ }^{1}$ Variable depending on the number of loadings and controls

${ }^{2}$ Cleaning of new glassware was accomplished by rinsing with DI water and drying. Used glassware was first soaked in a $2 \%$ to $5 \%$ cleaning solution of Contrad 70 prior to rinsing triply with DI water.

${ }^{3}$ Optional

${ }^{4}$ Instruments used to generate data for this SP are presented here. Refer to disclaimer on pg. 3.
} 
the water to $97{ }^{\circ} \mathrm{C}$ to $100{ }^{\circ} \mathrm{C}$ (or to a gentle boil). Optional: To expedite heating, the outer surfaces of the beaker can be wrapped in $\mathrm{Al}$ foil.

5.4 While heating, add the cleaned lengths of thread to the water. Add two threads to a beaker. So that the threads can be easily removed later and to avoid the creation of knots in the line, leave one end of each thread outside of the beaker and secure this with tape. Ensure that the thread is secure in its attachment because once exposed to the tape's adhesive, addition of that end of the thread to the solution/suspension could introduce contamination.

5.5 While the water is heating, prepare the following two solutions.

Silver nitrate $\left(\mathrm{AgNO}_{3}\right)$ solution: Into the first small beaker, add $0.36 \mathrm{~g}$ of $\mathrm{AgNO}_{3}$ into $20 \mathrm{~mL}$ of DI water to form a $0.11 \mathrm{~mol} \cdot \mathrm{L}^{-1}$ solution of $\mathrm{AgNO}_{3}\left(\left[\mathrm{Ag}^{+}\right]=0.11 \mathrm{M}\right)$. Ensure that the powder completely dissolves.

Citrate solution: In a second small beaker, add $0.48 \mathrm{~g}$ of trisodium citrate dihydrate $\left(\mathrm{Na}_{3} \mathrm{C}_{6} \mathrm{H}_{5} \mathrm{O}_{7} \cdot 2 \mathrm{H}_{2} \mathrm{O}\right)$ to $30 \mathrm{~mL}$ of DI water to form a $0.054 \mathrm{~mol} \cdot \mathrm{L}^{-1}$ solution of. Ensure that the solid completely dissolves.

5.6 When the water temperature is $>90{ }^{\circ} \mathrm{C}$, prepare a third solution containing sodium borohydride $\left(\mathrm{NaBH}_{4}\right)$. To a small beaker with $40 \mathrm{~mL}$ of DI water, add $0.40 \mathrm{~g}$ of $\mathrm{NaBH}_{4}$ to create a $0.26 \mathrm{~mol} \cdot \mathrm{L}^{-1}$ solution of $\mathrm{NaBH}_{4}$. Ensure that the powder completely dissolves.

5.7 Once the water reaches a slow steady boil record the temperature and increase stir speed so that the vortex formed in the center goes between $1 / 2$ to $3 / 4$ of the way to the bottom of the beaker. Steadily add your prepared solutions (steps 5.5 and 5.6) in the quantities indicated in Table 1 for validation thread 1. Use an Eppendorf pipette that is designed to work with the specified volumes. The following order of addition must be performed rapidly (ideally, $<10 \mathrm{~min}$ ). Complete steps 5.7.1 - 5.7.3 one loading concentration at a time:

5.7.1 First, add the citrate solution to the beaker. Ensure that the temperature is still $97{ }^{\circ} \mathrm{C}$ to $100{ }^{\circ} \mathrm{C}$ before proceeding.

5.7.2 Next add the Ag nitrate to the same beaker. Once this is added quickly record the temperature again and move on immediately to the final addition.

5.7.3 Drop-wise add in the sodium borohydride. It is important to ensure that the addition of this solution slowly especially for validation thread 1 , which has the greatest concentration of $\mathrm{Ag}^{0}$ (around $1 \mathrm{~mL} \cdot \mathrm{min}^{-1}$ ). For most of the intended $[\mathrm{AgNP}]$ in Table 1, addition of $\mathrm{NaBH}_{4}$ should immediately cause a color change from clear to dark yellowish/brown. By design, this reducing agent is added in excess and therefore should convert all $\mathrm{Ag}^{+}$to $\mathrm{Ag}^{0}$. NOTE: If $\mathrm{AgNO}_{3}$ concentrations are too high, aggregation may be unavoidable. 
5.7.4 Repeat steps 5.1.6.1-5.1.6.3 for the remaining validation threads. (Validation thread 6 is the positive control and is not treated with the three solutions.)

5.8 Allow the temperature to remain just below boiling for $\approx 30 \mathrm{~min}$ with stirring. After $30 \mathrm{~min}$ turn off the heat and stirring.

5.8.1 Optional: To obtain an approximation of the size and concentration of AgNPs that were formed in suspension, remove a $5 \mathrm{ml}$ aliquot from each, cool, and perform desired measurements (e.g., $U V$-vis, DLS, ICP-MS).

5.9 Cover the beaker with either a glass cover or parafilm and cool for $>12 \mathrm{~h}$. Shield all samples from light with $\mathrm{Al}$ foil to prevent photoreactions from occurring.

5.10 Once the suspension has cooled, fill a large beaker half way with DI water to wash loosely bound particles and/or aggregates from the AgNP-loaded threads.

5.11 While holding the thread ends, remove the tape from the side of the first container (the highest Ag concentration solution/suspension/loading). Aggregation may be visible in the beaker as sediment, floating material and/or as a coating on the thread, and the two threads may be tangled together. Lift the threads out of the beaker.

5.12 First, dip the threads into the clean DI water and move them in a circular motion in order to wash the threads. Ensure that the entirety of the coated thread is submerged with the exception of the section that was taped/out of the suspension. The loose aggregates may be removed and loosely attached AgNPs may become detached. As a result, the DI water may change to a light shade of yellow and/or have visible aggregates floating in the cleaning water.

5.13 If the threads were tangled, untangle them and repeat step 5.1.10.

5.14 Hang each thread to air dry. This can be done by fixing the previously taped end (presumably Ag free) to a horizontal bar. Make sure that the drying lengths of the thread do not touch anything, including each other.

5.15 Properly dispose of wash water as nanoparticle aqueous waste.

5.16 Repeat steps 5.10 to 5.15 separately for validation threads 2, 3, 4, 5, and 6 (see Table 1). Complete all steps for one thread number before proceeding to the next thread number.

5.17 Once washing is completed and the threads are dried, remove the hanging threads from their drying location. Cut off the end of the thread that was taped/out of suspension to ensure no contamination is transferred to the final product. For example, if $2.5 \mathrm{~cm}$ was held by the tape, then $2.5 \mathrm{~cm}$ or more of that end should be removed from the final product. This end should be properly disposed of as nanoparticle waste. 
5.18 Use gloves or tweezers to move the threads into containers; validation threads with different numbers must be stored in different containers so as to prevent cross contamination which could skew future measurements of the surface concentrations.

5.18.1 Figure 1 (A) provides photographs of the typical appearance of the validation threads, listed by their estimated $\left[\mathrm{Ag}^{0}\right]$ from Table 1 . Estimated $\left[\mathrm{Ag}^{0}\right]$ are based on $100 \%$ conversion to metallic AgNP in suspension, zero loss of Ag to adsorption or aggregation, and zero loss of volume due to the evaporation. As a result of these assumptions (especially the zero-loss due to evaporation), it is emphasized that the reported 'estimated $\left[\mathrm{Ag}^{0}\right]$ ' are estimates of suspensions for internal comparison and should not be considered quantitatively accurate for validation threads.

5.19 Store the threads away from light until needed for characterization. When smaller sections of a validation thread are cut for samples, the sections should be stored in the same manner unless reported otherwise.

\section{Procedures for Measurement of Threads}

6.1 AFM: AFM was employed to image micrometer-scale areas of AgNP-loaded threads using tapping mode. To immobilize a thread for measurement, single fibers were removed from the thread and pinned on glass slides with epoxy glue at the thread ends. Prior to scanning, the AFM probe used was visually guided under the optical microscope of the AFM to land the tip on the target fiber. The acquired AFM topography images were flattened in the second order to account for the roundness of the fiber.

6.2 FE-SEM: An additional set of AgNP loaded validation threads were freshly prepared to examine the potential of confirming the presence of nanoparticles using FE-SEM. About 1 $\mathrm{cm}$ lengths of the threads were cut and attached to an SEM specimen holder using doublesided carbon tape. To minimize charging and increase magnification of the surface, selected threads were sputter-coated with a layer of gold no more than $10 \mathrm{~nm}$ in thickness using a Denton Vacuum Desktop III system.

6.3 EDS: Elemental compositions were determined from spectra acquired at acceleration voltages of $10 \mathrm{kV}$ and $20 \mathrm{kV}$. Thread samples were adhered to double-sided copper tape and spectra were acquired without additional coatings. Weight percentages were calculated for all detected elements using commercially available software.

6.4 ICP-MS: ICP-MS was used to measure the total silver in the prepared threads. Three subsamples per loaded thread of approximately equal length were prepared for analysis by cutting with clean Teflon scissors; except for the control, where only one sub-sample was processed. The mass of each analyzed sub-sample was measured on a calibrated Mettler AT 20 analytical balance. Sub-sample masses ranged from nominally $1.6 \mathrm{mg}$ to $2.3 \mathrm{mg}$. Samples were placed in clean 50-mL Perfluoroalkoxy (PFA) Teflon beakers with $1 \mathrm{~g}$ concentrated nitric acid $\left(\mathrm{HNO}_{3}\right)(\mathrm{Optima}$ grade, Thermo Fisher Scientific, Waltham, MA, USA), covered and refluxed on a hot plate at high heat for $40 \mathrm{~min}$. An additional $1 \mathrm{~g} \mathrm{HNO}_{3}$ was added and samples were refluxed for an additional $40 \mathrm{~min}$. The thread was observed to completely dissolve. Samples were taken to dryness, re-dissolved in $2 \%$ volume fraction $\mathrm{HNO}_{3}$, and quantitatively transferred to clean, tared, low-density polyethylene bottles, forming the first serial dilution. After the addition of nominally $2 \mu \mathrm{g} / \mathrm{kg}$ indium (In) internal standard, samples were quantitatively diluted a second time, to an analysis concentration of nominally $5 \mu \mathrm{g} / \mathrm{kg} \mathrm{Ag}$. 
From the mass spectrometric analyses, the mass fraction of Ag in each sample was calculated from the using an external calibration curve. Temporal changes in signal intensity resulting from effects of the cotton thread were corrected via the In internal standard.

6.5 XPS: Threads were mounted so as to be elevated to minimize contributions to the spectra from the sample holder. Presented spectra were acquired using monochromatic Al Ka Xrays acquired at $40 \mathrm{eV}$ pass energy over a variety of spot sizes, up to $250 \mu \mathrm{m}$. Weight percentages were calculated from atomic percentages that were based on the elemental relative sensitivity factor (RSF) corrected areas for carbon, oxygen, silicon and silver. RSFs were obtained from the instrument manufacturer.

\section{Outcomes}

7.1 Visible observations can be quite instructive as a starting point regarding whether or not the cotton threads have been successfully loaded with AgNPs (see Figure 1(A)). Specifically, the characteristic yellow/brown color representative of AgNP suspensions (see Figure 1(B) as an example from a separate study) is observed on the threads in varying shades, from a dark yellow/brown in validation thread 1 to a faint yellow for validation threads 4 and $5[3$, 4]. This should be especially evident since a white thread was employed (see control in Figure 1 (A)). Validation thread 1 appeared yellow/brown after washing even though aggregation occurred suggesting a highly localized reaction.

\subsection{Microscopy based measurements}

7.2.1 AFM measurements provided evidence of particulate deposition on the surface of the thread fibers as demonstrated by comparing the images shown in Figure 2(A) and 2(B) for zero-Ag control and the highest Ag loading, respectively. After image processing, the control in Figure 2(A) was found to be devoid of any particles while Figure 2(B) appeared to have a significant fraction of particles and aggregates with heights measuring between $20 \mathrm{~nm}$ and $40 \mathrm{~nm}$. While AFM is quite useful and successful at identifying nanoparticles on the Ag-loaded threads (data not shown for the remaining threads), it is important to note that nanoscale features were not found at every analysis point and required some searching.

7.2.2 SEM images were acquired on three samples that were from a small batch of Ag loaded cotton threads prepared separately from those listed in Table 1 and presented in Figure 1(A). Figure 3 demonstrates the morphological characteristics of the individual fibers. As can be observed, the fibers transform from being smooth and devoid of features in their zero-loading state (control thread) to increasingly irregular with large adsorbed particulates with increasing estimated $\left[\mathrm{Ag}^{0}\right]$. Figure 4 demonstrates higher resolution images for the $7.7 \times 10^{-4} \mathrm{~mol} \cdot \mathrm{L}^{-1}\left[\mathrm{Ag}^{0}\right]$ samples only. Figure $4(\mathrm{~A})$ demonstrates the presence of nanoscale particles on the surface of this thread in addition to features/aggregates $>100 \mathrm{~nm}$ in size. Figure 4 (B) reveals the presence of particles from $20 \mathrm{~nm}$ and greater in diameter with the alleviation of charging effects by a gold coating less than $10 \mathrm{~nm}$ in thickness. It was not possible to differentiate between particles smaller than $20 \mathrm{~nm}$ and the gold coating, which is known to produce nanoparticles on the 
order of $10 \mathrm{~nm}$ and less. However, the presence of nanoscale features in Figure 4(A) and EDS spectra (data not shown), which revealed the presence of Ag on the thread's surface, suggested that the nanoparticles $>20 \mathrm{~nm}$ observed in Figure 4 (B) were indeed AgNPs.

\subsection{Spectroscopy based measurements}

7.3.1 ICP-MS measurements demonstrated an increase in the total measured Ag concentration (see Table 2) with increased estimated $\left[\mathrm{Ag}^{0}\right]$; the results are plotted in Figure 5(A). The observed variability between subsamples for the highest four Ag loadings (validation threads 1 to 4 ) is greater than the expected variability of the measurement method (5\%, relative), indicating some heterogeneity in the Ag content along the length of a thread.

7.3.2 XPS measurements demonstrated an increase in the surface Ag content with increased estimated $\left[\mathrm{Ag}^{0}\right]$, (see Figure 6). The Ag loading increased from zero Ag measured on the control thread to a mass percentage of $8.5 \% \pm 1.2 \%$ (average and range of 2 points) for validation thread 1 (highest Ag loading, Figure 5(B)). On average, surface Ag concentration increased with increasing estimated $\left[\mathrm{Ag}^{0}\right]$, although some thread loadings revealed a larger degree of heterogeneity than others. XPS measurements suggested a surface enhancement for a given validation thread when compared to ICP-MS.

7.3.3 EDS Measurements were consistent with both XPS and ICP-MS measurements, with the measured Ag concentration on the thread increasing with increased estimated $\left[\mathrm{Ag}^{0}\right]$ (See Figure 5(C)). In further support for a surface enhancement of Ag deposition, there was a clear increase in the measured Ag weight percentage at lower accelerating voltages, which one would expect if AgNPs were adsorbed at higher concentrations on the thread surfaces.

\subsection{Discussion of Outcomes}

Several different microscopy-based techniques have been employed in conjunction with spectroscopic and chemical analysis to provide evidence of AgNPs loaded onto the surface of the cotton threads. Both AFM and SEM images revealed the presence of particles loaded onto the threads with dimensions at the nanoscale, in addition to other aggregates/ agglomerates/features that were of larger size.

Ag-loaded cotton threads consistently had a significantly greater mass $\%$ of Ag measured at the surface as observed by comparing XPS and ICP-MS measurements (Figure 5(A) and 5(B)). This finding was further corroborated by EDS measurements (Figure 5(C)) which yield Ag mass \% values typically falling between the XPS and ICP-MS values. This suggests that the Ag was predominantly adsorbed via reduction on or near the cotton fiber's surface as opposed to absorption and reduction of Ag within the bulk of the thread. This conclusion is supported by Figure 7, which is a plot of Ag concentration in the threads measured by XPS vs that measured by ICP-MS. The two techniques show good correlation over all but the highest concentration ranges measured, as indicated by the upper solid line representing a linear relationship. The lower dashed line in Figure 7 is representative of identical surface and bulk compositions; data lying above this line indicates a surface enhancement. As is clearly depicted by the upper solid line in Figure 7, a surface 
enhancement factor of approximately 30 is what was observed for the four lower estimated $\left[\mathrm{Ag}^{0}\right]$ values.

\section{Abbreviations}

$\begin{array}{ll}\text { AFM } & \text { Atomic Force Microscopy } \\ \text { AgNP } & \text { Ag nanoparticle } \\ \text { DI } & \text { Deionized } \\ \text { EDS } & \text { Energy Dispersive X-ray Spectroscopy } \\ \text { eV } & \text { electron volt } \\ \text { FE-SEM } & \text { Field Emission Scanning Electron Microscopy } \\ \text { ICP-MS } & \text { Inductively Coupled Plasma Mass Spectrometry } \\ \text { Nano-EHS } & \text { Environmental, Health and Safety as it pertains to nanomaterials } \\ \text { NNI } & \text { National Nanotechnology Initiative } \\ \text { UV-vis } & \text { Ultraviolet Visible Spectroscopy } \\ \text { Wt\% } & \text { Weight percent } \\ \text { XPS } & \text { X-ray Photoelectron Spectroscopy }\end{array}$

\section{Acknowledgements}

We would like to thank Debra Kaiser for her direction and vision in the characterization of nanomaterials in consumer products, from which the development of this protocol derived.

\section{References}

1. National Nanotechnology Initiative: Environmental, Health and Safety Research Strategy. 2012, Presidents Council of Advisors on Science and Technology: National Science and Technology Council Committee on Technology: Washington, DC.

2. Wijnhoven, S.W.P., et al., Nano-silver - a review of available data and knowledge gaps in human and environmental risk assessment. Nanotoxicology, 2009. 3(2): p. 109-138.

3. Gorham, J.M., et al., UV-induced photochemical transformations of citrate-capped silver nanoparticle suspensions. Journal of Nanoparticle Research, 2012. 14(10).

4. Gorham, J.M., et al., Storage Wars: how citrate-capped silver nanoparticle suspensions are affected by not-so-trivial decisions. Journal of Nanoparticle Research, 2014. 16(4).

5. MacCuspie, R.I., et al., Just add water: reproducible singly dispersed silver nanoparticle suspensions on-demand. Journal of Nanoparticle Research, 2013. 15(7).

6. Benn, T.M. and P. Westerhoff, Nanoparticle Silver Released into Water from Commercially Available Sock Fabrics. Environ. Sci. Technol., 2008. 42: p. 4133-4139.

7. ASTM, E2456-06(2012): Standard terminology relating to nanotechnology. 2012.

8. ISO, IS TS 80004-1:2010: Nanotechnologies-Vocabulary-Part 1: Core Terms. 2010, ISO: Geneva, Switzerland.

9. Wikipedia. http://en.wikipedia.org/wiki/Textile. 
Figures and Tables for SP

\begin{tabular}{|c|c|c|c|c|}
\hline \multirow{2}{*}{$\begin{array}{c}\text { Validation Thread } \\
\text { Number }\end{array}$} & \multicolumn{3}{|c|}{ Starting Materials $(\mathbf{m L})$} & \multirow{2}{*}{$\begin{array}{c}\text { Estimated }\left[\mathbf{A g}^{\mathbf{0}}\right] \\
\left(\mathbf{m o l} \bullet \mathbf{L}^{\mathbf{- 1}}\right)\end{array}$} \\
\cline { 2 - 5 } & $\mathbf{A g N O}_{\mathbf{3}}$ & $\mathbf{N a}_{\mathbf{3}} \mathbf{C}_{\mathbf{6}} \mathbf{H}_{\mathbf{5}} \mathbf{O}_{\mathbf{7}}$ & $\mathbf{\mathbf { N a B H } _ { \mathbf { 4 } }}$ & $3.60 \mathrm{E}-03$ \\
\hline 1 & 6.25 & 12 & 5.2 & $7.20 \mathrm{E}-04$ \\
\hline 2 & 1.11 & 2.125 & 0.93 & $1.84 \mathrm{E}-04$ \\
\hline 3 & 0.278 & 0.53 & 0.23 & $3.73 \mathrm{E}-05$ \\
\hline 4 & 0.056 & 0.11 & 0.047 & $1.87 \mathrm{E}-05$ \\
\hline 5 & 0.028 & 0.053 & 0.025 & 0 \\
\hline 6 & 0 & 0 & 0 & \\
\hline
\end{tabular}

Table 1: The volumes of stock materials necessary to create different validation threads. The concentrations of the stock starting materials are outlined in steps 5.1.4 and 5.1.5 of the procedure. Estimated $\left[\mathrm{Ag}^{0}\right]$ is based on no loss of $\mathrm{Ag}$ in suspension due to adsorption, complete reduction of $\mathrm{Ag}^{+}$and no evaporation. Therefore, estimated $\left[\mathrm{Ag}^{0}\right]$ values should only be used for internal comparisons and not as an accurate representation of concentrations. 

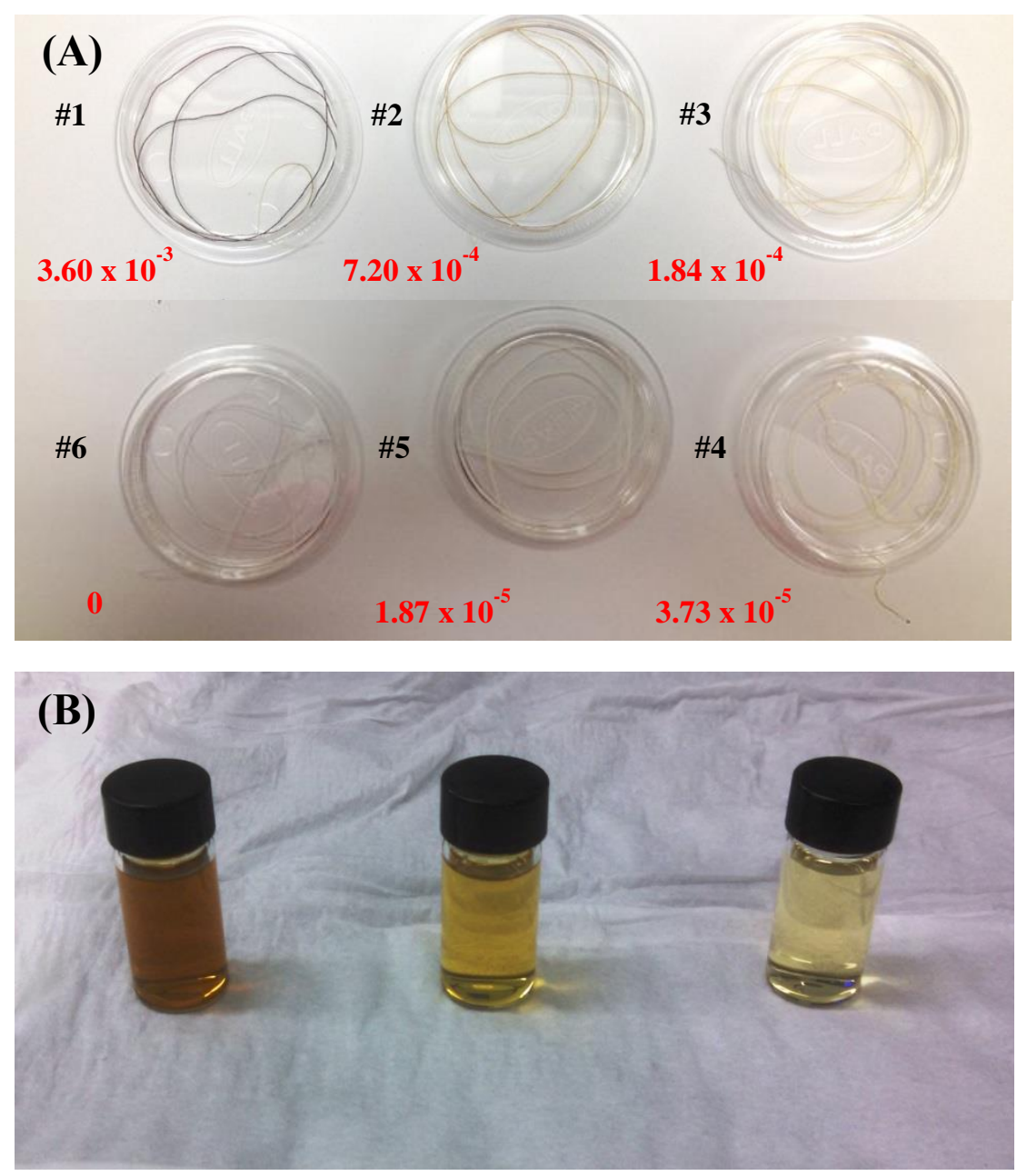

Figure 1: (A) Example of cotton threads prepared with different loadings of AgNPs. Additionally, 1 control sample with no $\mathrm{Ag}$ added is included. Samples go from a dark yellow/brown to near white and are labelled according to the mol $\bullet \mathrm{L}^{-1}$ estimates and thread \# listed in Table 1 . The control remained white. (B) Examples of a series dilution of a AgNP suspension color for (left to right) $\left(7.7 \times 10^{-4}, 1.93 \times 10^{-4}\right.$, and $\left.3.85 \times 10^{-5}\right)$ estimated $\left[\mathrm{Ag}^{0}\right]\left(\mathrm{mol} \bullet \mathrm{L}^{-1}\right)$. 

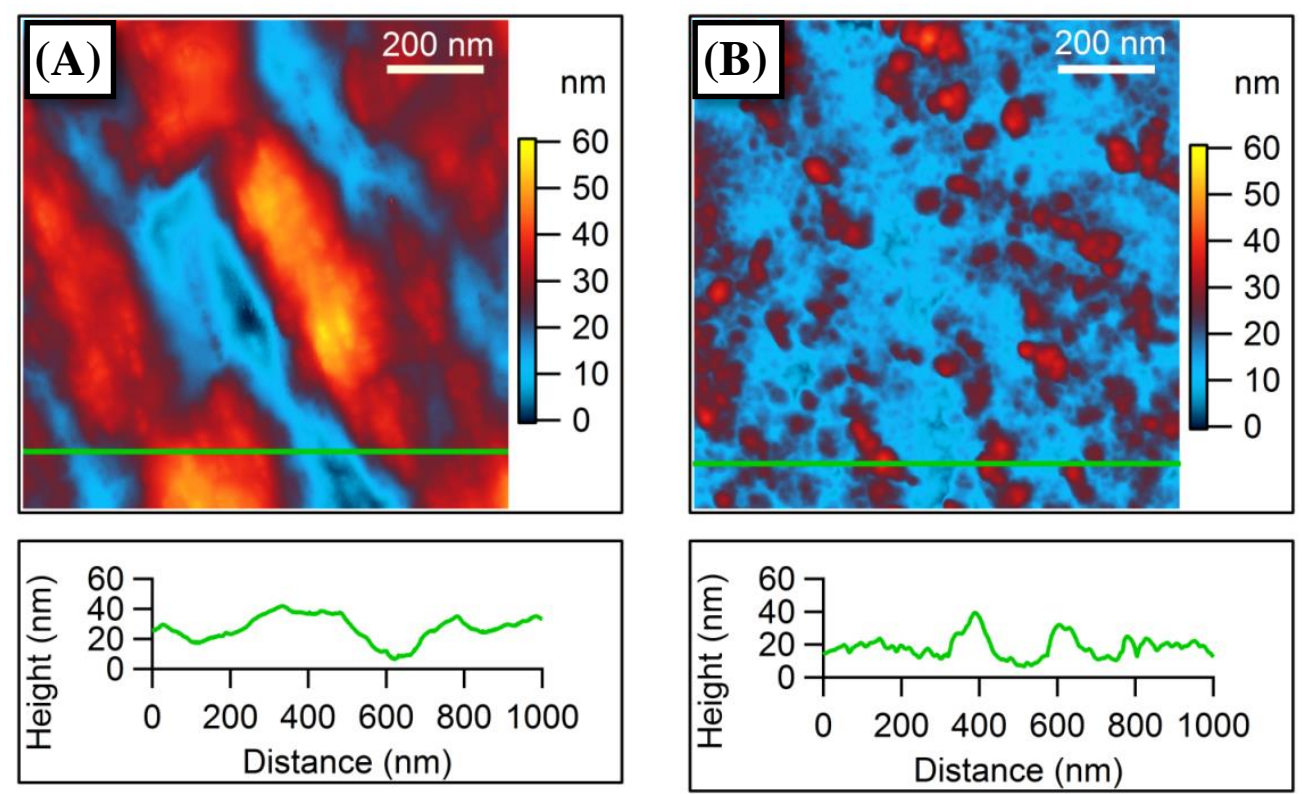

Figure 2: AFM micrographs of thread surfaces. (A) A fiber from validation thread 6, the control, with no evidence of particles adsorbed to the surface and (B) a fiber from the preparation of the highest $\mathrm{Ag}$ concentration, validation thread 1, with evidence for isolated, nanoscale particles and aggregates on its surface. 

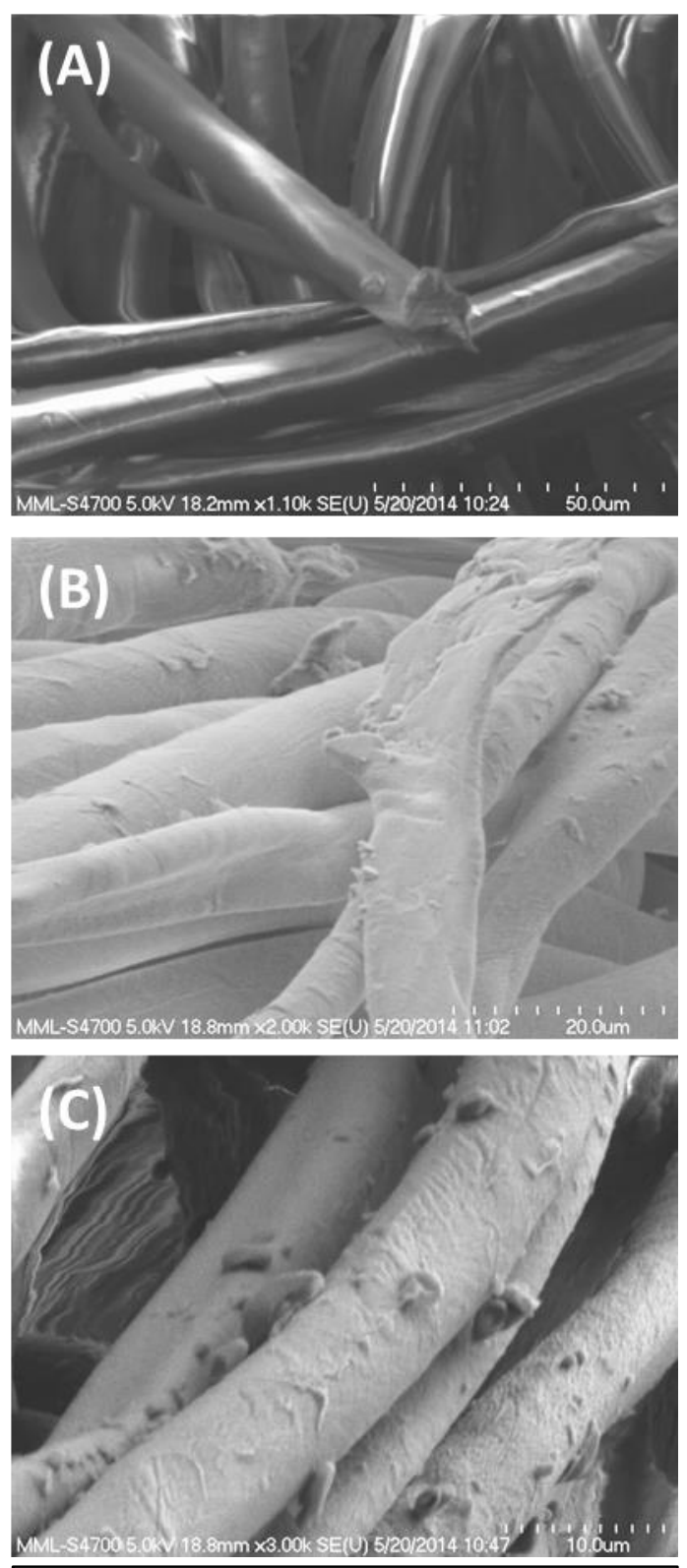

Figure 3: Images of three, as prepared cotton threads. Fibers were freshly prepared in at estimated $\left[\mathrm{Ag}^{0}\right]$ of (A) validation thread $6,(\mathrm{~B})$ an intermediate Ag loading $\left(1.97 \times 10^{-4} \mathrm{~mol} \cdot \mathrm{L}^{-1}\right)$ and $(\mathrm{C})$ a high $\mathrm{Ag}$ loading $\left(7.7 \times 10^{-4} \mathrm{~mol} \bullet \mathrm{L}^{-1}\right)$. 

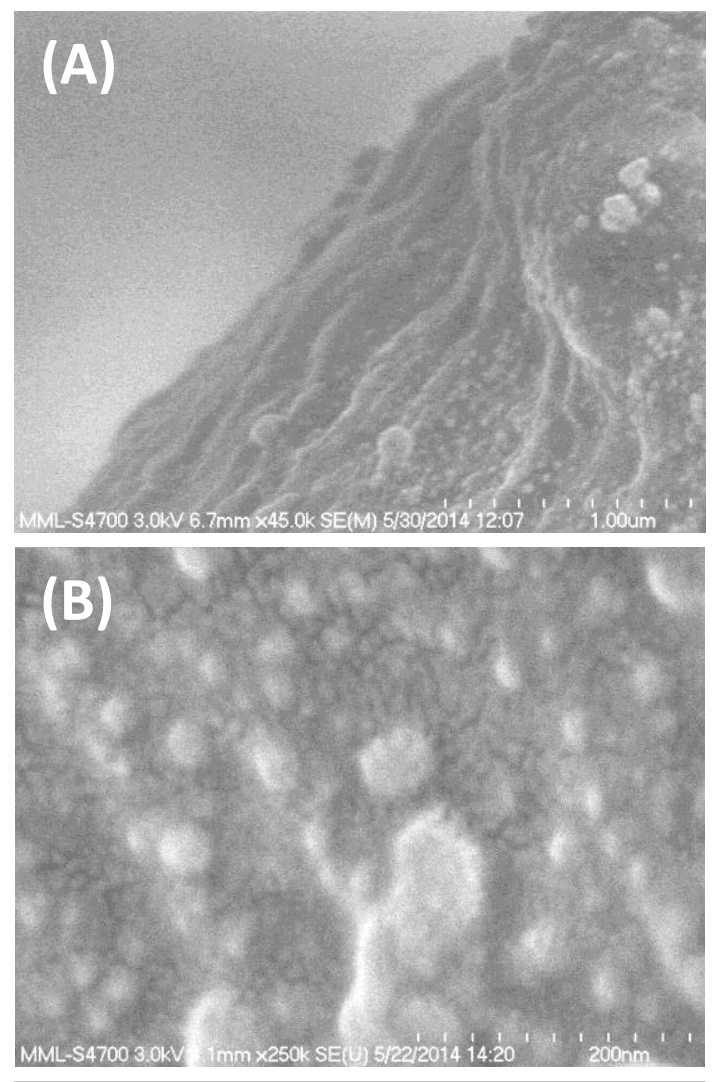

Figure 4: Two SEM images of a $7.7 \times 10^{-4} \mathrm{~mol} \cdot \mathrm{L}^{-1} \mathrm{Ag}$ loaded cotton thread. (A) An image taken as prepared and (B) an image taken with a layer of gold, no more than $10 \mathrm{~nm}$ thick, to dissipate charging. Particles with sizes $<100 \mathrm{~nm}$ were present in both images, providing further evidence of $\mathrm{Ag}$ nanoparticle deposition on the surface of the cotton fibers. 


\begin{tabular}{|c|c|c|c|}
\hline \multirow{2}{*}{$\begin{array}{c}\text { Validation Thread } \\
\text { Number }\end{array}$} & Estimated $\left[\mathrm{Ag}^{\mathbf{0}}\right.$ & Measured Ag & \\
\hline & $\mathrm{mol} \cdot \mathrm{L}^{-1}$ & ICP mass\% & S.D \\
\hline 1 & $3.60 \mathrm{E}-03$ & 3.1 & 2.4 \\
\hline 2 & $7.20 \mathrm{E}-04$ & 0.142 & 0.02 \\
\hline 3 & $1.84 \mathrm{E}-04$ & 0.0365 & 0.0089 \\
\hline 4 & $3.73 \mathrm{E}-05$ & 0.0219 & 0.0027 \\
\hline 5 & $1.87 \mathrm{E}-05$ & 0.01258 & 0.0005 \\
\hline 6 & 0 & 0.0001 & N/A \\
\hline
\end{tabular}

Table 2: ICP-MS measurements and standard deviations for three sub-samples per level of the AgNP loaded threads and one sample of the control. 


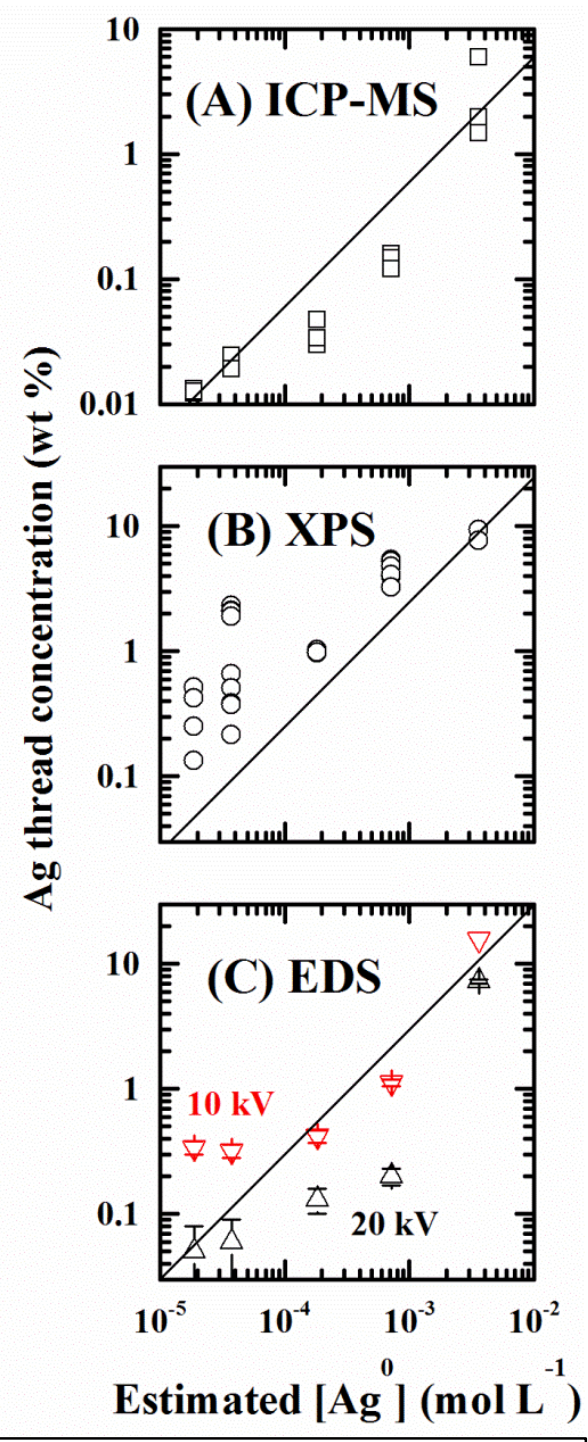

Figure 5: Plots of measured $\mathrm{Ag}$ concentration on exposed cotton threads as a function of estimated $\left[\mathrm{Ag}^{0}\right]$. (A) ICPMS measured total $\mathrm{Ag}$ in and on the thread; (B) XPS measured surface concentration on the thread; and (C) EDS measures predominantly surface $\mathrm{Ag}$, with a depth sensitivity that increases with accelerating voltage (shown). The straight lines are guides to the eye indicating linear relationships. 


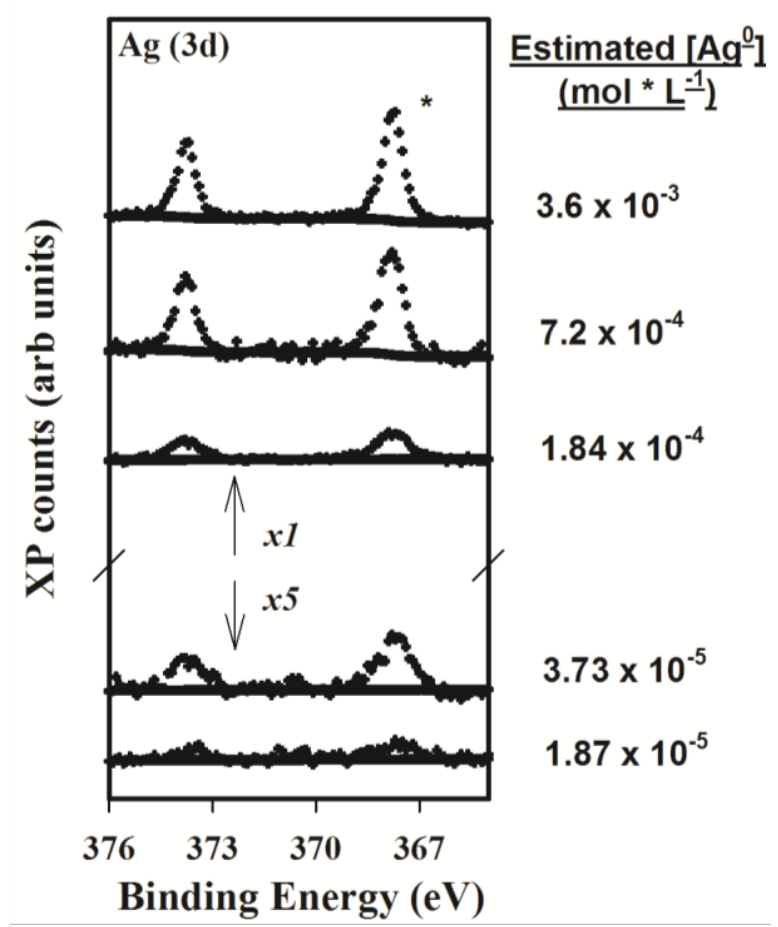

Figure 6: XPS spectra of the $\mathrm{Ag}(3 \mathrm{~d})$ region for the five cotton threads loaded with AgNP. *XPS acquisition for validation thread 1 collected photoelectron intensity from roughly $1 / 4$ the area, acquiring $1 / 4$ the signal, as compared to the remaining fibers. 


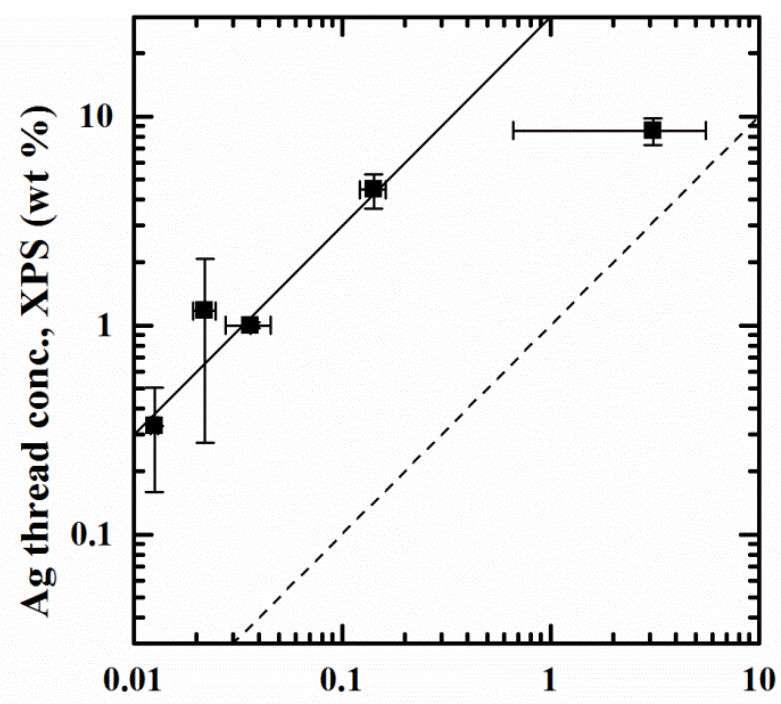

Ag thread conc., ICP-MS (wt \%)

Figure 7: Plot of Ag concentration measured by (surface sensitive) XPS vs (bulk sensitive) ICP-MS. The straight lines are guides to the eye, representing linear relationships. The lower, dashed line represents identical surface and bulk concentrations; the data suggest significant surface enhancement. Average and standard deviation is representative of three samples measured by ICP-MS and $\geq 2$ spots by XPS. 